\title{
THE THERMAL HISTORY AND STRUCTURE OF COMETARY NUCLEI
}

\author{
HANS RICKMAN \\ Astronomiska Observatoriet \\ Box 515 \\ S-751 20 Uppsala \\ Sweden
}

ABSTRACT. Cometary nuclei are often considered to be the most primitive bodies of the solar system. Thus it is particularly important to find out what structural changes may have been brought about as a result of their thermal evolution. Is there reason to believe that the bulk composition of the nucleus of a present-day short-period comet may differ from that of the original planetesimal in the solar nebula? Apart from the development of a non-volatile surface layer ('dust mantle'), what further depth-dependent differentiation can we expect in such a nucleus? These are the ultimate questions addressed in this paper, and attention is focused on the two most active stages of thermal evolution: the early planetesimal stage with internal heating basically due to radioactive decay, and the recent or present cometary phase with strong external heating due to insolation of the surface.

Recent developments described in this review include consideration of more remote sites of cometary formation, attention to a porous structure over a wide range of scales, likelihood of an initially amorphous phase of the $\mathrm{H}_{2} \mathrm{O}$ ice, laboratory data on gas trapping and transport phenomena in amorphous ice, reevaluation of the rôle of clathrate hydrates, calculation of subsurface migration and latent heat transport by volatiles, and refinement of global heat flow models for cometary nuclei with internal heat sources.

\section{Introduction}

Cometary nuclei are usually viewed as remnants of the planetesimal population of the outer parts of the solar nebula. Hence, these bodies are potential tracers of nebular processes and the growth of planetary bodies, and this has led to a vivid interest in the question of the cometary counterpart of geochemical evolution: How much and in what sense has a cometary nucleus evolved since it formed? By astronomical remote sensing techniques, or indeed any spaceborne experiment currently envisaged, we are restricted to information on the outermost layers of the nucleus. Whether this tells us anything about the primordial composition and structure of the whole nucleus, and if so, how the observed information is to be interpreted, obviously depends on the past thermal and chemical evolution.

To characterize this thermal history, we have to draw upon astrophysical concepts of cometary formation and the constitution of cometary material, laboratory results on the behavior of such material upon warming, and theoretical models predicting how the nucleus structure responds to this. Very significant progress has been achieved in all these respects 
during the $1980 \mathrm{~s}$, and one can hence make much more relevant statements now than earlier. Relevant background information for the following discussion can be found in recent reviews dealing with the composition of comets (Weaver 1988) and their cosmogonic interpretation (Geiss 1987, Rickman and Huebner 1990). Furthermore, the physical evolution of comets has been reviewed by Weissman (1986a), Rickman (1987), and Weissman and Stern (1990), and modelling of the structure and evolution of cometary nuclei by Houpis (1990) and Prialnik (1989). This review will aim for a comprehensive discussion of all these aspects in order to summarize the present state of the art. However, it is advisable to keep in mind that the topic is in rapid development and that there is nothing definitive about the present picture. Some of the tentative conclusions that can now be formulated may soon become outdated in the light of new discoveries.

One easily identifies three different phases of the evolution in question: firstly, a quick and vigorous phase (duration $\sim 10^{6}-10^{8} \mathrm{yrs}$ ), connected with the formation of the comets; secondly, a very long (several times $10^{9} \mathrm{yrs}$ ) and relatively quiescent Oort cloud phase; and finally, the drastic rearrangements occurring upon approaches close to the Sun. This last phase is expected to span a period of $\lesssim 10^{6}$ yrs. These three phases will be dealt with in turn, but before this we have to consider the physical and chemical processes influencing cometary nuclei in more general terms.

\section{Amorphous and Porous Ices}

If one imagines that comets formed in a cooling solar nebula, then it is natural to expect that $\mathrm{H}_{2} \mathrm{O}$ ice condensed in the crystalline state at temperatures around $150 \mathrm{~K}$, somewhat depending on the ambient pressure (see, e.g., Lewis and Prinn 1980). The ice thus formed would remain crystalline even if the temperature would afterward fall much lower. However, if the condensation occurred as a result of the compaction of a collapsing interstellar cloud at the onset of solar system formation, then the temperature should have been far below $100 \mathrm{~K}$, and the ice would form in the amorphous state (Klinger 1980, 1981, Smoluchowski 1981; the first suggestion of amorphous ice playing a rôle in cometary behavior is due to Patashnick et al. 1974). This form of ice is not absolutely stable, and upon heating, it crystallizes with the liberation of latent heat to the amount of 90 $\mathrm{J} / \mathrm{g}$ (Ghormley 1968). The rate of crystallization should be expressible as an exponential function of temperature $T$ (Rickman et al. 1985):

$$
t_{\mathrm{cr}}=t_{*} \cdot \exp \left(T_{*} / T\right)
$$

where $t_{\mathrm{cr}}$ is the crystallization time. Laboratory results displayed in Fig. 1 (Schmitt et al. 1989) have verified this expectation, yielding the values $t_{*}=2.65 \cdot 10^{-17}$ hours, and $T_{*}=5370 \mathrm{~K}$. Unfortunately, one needs a wide extrapolation from the typical timescales attained in laboratory experiments to those relevant to comet formation, but with some confidence one can say that amorphous ice could survive for millions of years or more at temperatures below $85 \mathrm{~K}$. Thus, considering that time scales from only $10^{4}$ yrs (Weidenschilling 1980 ) up to $10^{6}$ to $10^{7}$ years (e.g., Shul'man 1983) have been estimated 
for comet formation by accretion of grains in the solar nebula, and that estimates of the nebula lifetime are generally $\sim$ several times $10^{6}$ yrs (Cameron 1985, Morfill et al. 1985), the nuclei should have formed in the amorphous state if the region of formation was colder than $85 \mathrm{~K}$.

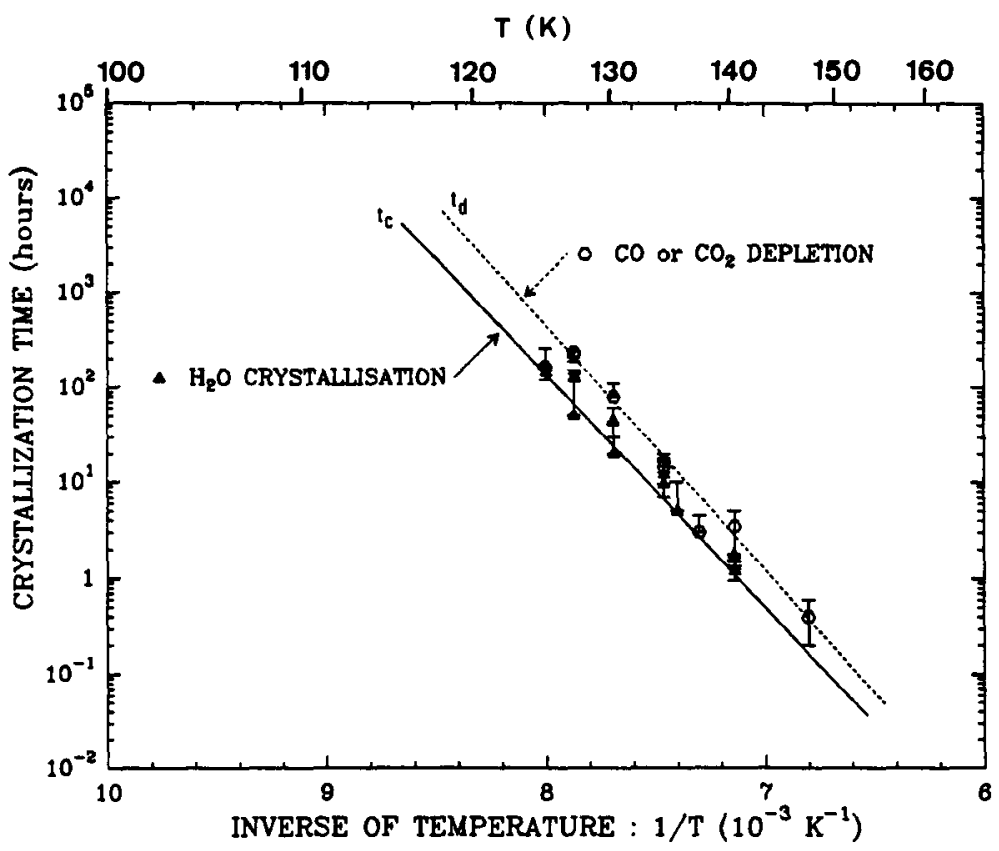

Figure 1. Time scales of crystallization of amorphous $\mathrm{H}_{2} \mathrm{O}$ ice (solid line) and loss of more volatile species $\left(\mathrm{CO}\right.$ or $\left.\mathrm{CO}_{2}\right)$ as functions of the inverse of the temperature, according to Schmitt et al. (1989). Measurements, represented with error bars, have been performed in the temperature range from 125 to $150 \mathrm{~K}$.

One cannot be sure, a priori, that this must have been the case. However, for the relatively remote sites of cometary origin near Neptune's orbit or beyond that have recently been favored (e.g., Fernández and Ip 1981, Greenberg 1983, Yamamoto and Kozasa 1988), it seems very likely. Furthermore, as will be shown below, the observed volatile composition of comets may indeed give some firm evidence for the existence of amorphous ice in comets. Such ice has been produced in the laboratory by condensing water vapor onto a cold plate under near-vacuum conditions. It is experimentally found to be extremely porous - Laufer et al. (1987) liken the structure of this solid to a woolen carpet (see also Schmitt 1986). This may be referred to as a micro-porosity characteristic of each individual grain making up the comet.

In addition to this, the grains could be expected to accrete into an open structure where voids contribute a large fraction of the volume. This has been argued by Donn and Rahe (1982), and it is a distinctive feature of the fractal structure proposed by Daniels and 
Hughes (1981) and Donn and Hughes (1985) to result from random accretion. One will then be dealing with pores and voids of a wide range of dimensions, from those of single grains to those of large chunks or 'cometesimals' (Weissman 1986b). All this can be referred to as macro-porosity.

It has been suspected for some time (e.g., Wallis and MacPherson 1981) that the nongravitational accelerations measured for some short-period comets imply a fairly low density for the nuclei, and if this is the case, then a substantial porosity is also implied. Recent analyses of comet P/Halley (Rickman 1986, 1989; Sagdeev et al. 1988) have tended to substantiate this suspicion, though Peale (1989) argued for a higher density with very large error bars. Furthermore, the very low densities of some cometary meteor particles, interpreted by Greenberg (1986) in terms of the bulk composition of his 'interstellar' model, mean that the cometary material from which these particles originate must be very porous as well (see also Greenberg and Grim 1986). Hence, there is some observational support for the belief that cometary nuclei are indeed very porous objects. This view has far-reaching consequences for the nuclei's thermal behavior:

1) It opens the possibility for gas diffusion inside the nucleus, so that if there is a constituent that is more volatile than the predominant $\mathrm{H}_{2} \mathrm{O}$ and that vaporizes in the interior, then this vapor can diffuse to the surface and contribute to the gas production of the comet. It can also diffuse downward and perhaps recondense at colder layers. The result of such a transport, if it can indeed act on a large scale, is a chemical differentiation of the comet.

2) The gas carries its latent heat of sublimation, and since this heat is released by recondensation at a colder place, we are dealing with a heat transfer process playing a rôle similar to that of conduction. It is referred to as advection. When gas diffuses toward warmer layers, it exchanges heat with the warmer solid material, which is hence cooled by the gas. Again, we have a process tending to reduce the thermal gradient, and this is sometimes called convection (using a somewhat ambiguous term).

\section{Thermal Behavior of Cometary Material}

\section{THE ICE COMPONENT}

What thermal properties can we expect from cometary material? In particular, can we estimate the relevant values of the thermal conductivity and diffusivity? Interestingly, amorphous ice has a drastically different thermal conductivity than crystalline ice (Klinger $1980,1981)$. While the conductivity of crystalline ice goes inversely with temperature (see below), that of amorphous ice varies as the heat capacity, increasing linearly with temperature (Klinger 1980). At $T \sim 150 \mathrm{~K}$, the difference is an order of magnitude in favor of the crystalline conductivity, and with decreasing temperature, it grows even larger.

At face value, this difference might lead to very different behavior in the early stages of cometary evolution, since a crystalline nucleus and an amorphous one might respond quite differently to heating at low temperatures. However, we must also consider that the bulk 
conductivity of compact material is affected by porosity and supplemented by gas-phase conductivity. Porosity, of course, reduces the conductivity $(K)$, and this reduction can be expressed by Russell's formula (see Espinasse 1989):

$$
K=K_{\text {comp }} \cdot \frac{\left(1-p^{2 / 3}\right)}{\left(1-p^{2 / 3}+p\right)}
$$

where $p$ is the porosity and $K_{\text {comp }}$ is the conductivity of compact material. Considering the density estimates for Halley's comet (Rickman 1989), it is thus reasonable to expect a decrease to $20 \%$ to $30 \%$ of $K_{\text {comp }}$.

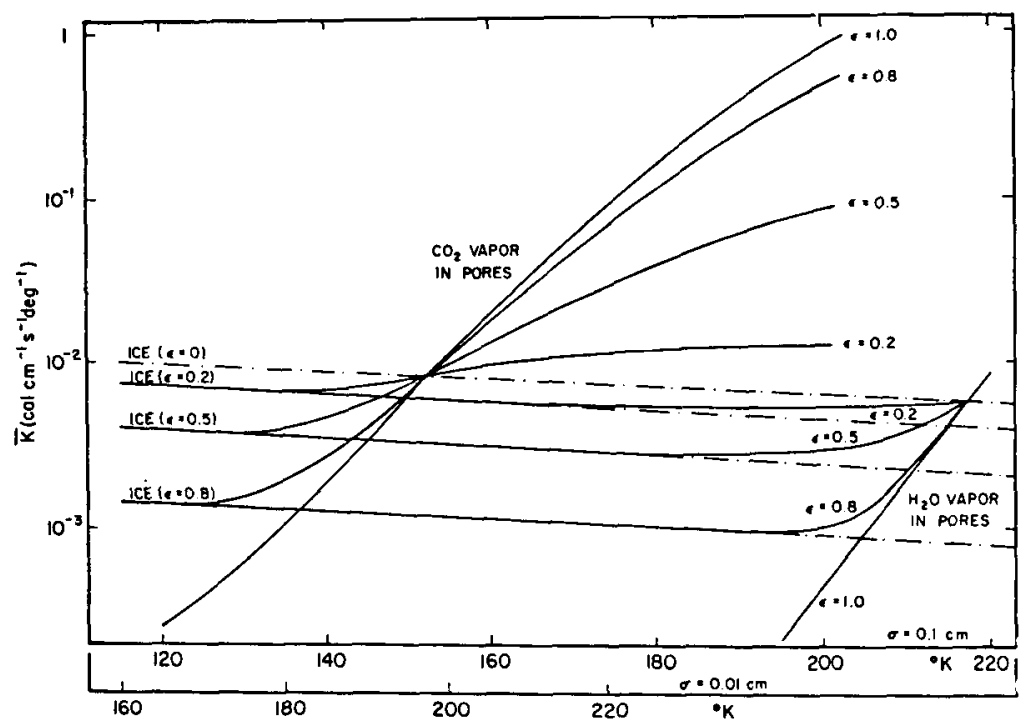

Figure 2. Effective thermal conductivity of porous ices as a function of temperature, according to Smoluchowski (1982). The different temperature scales correspond to different pore sizes $(\sigma)$, and the different curves refer to different porosities $(\epsilon)$.

Unfortunately, gas-phase conductivity is much more difficult to quantify, since it depends on the detailed chemical composition of the material and its previous history. Smoluchowski $(1982,1985)$ first drew attention to the possible importance of such conductivity for cometary nuclei, and Fig. 2 shows his estimated conductivities as functions of temperature both for pure $\mathrm{H}_{2} \mathrm{O}$ ice and for a $\mathrm{H}_{2} \mathrm{O}$ ice with an admixture of $\mathrm{CO}_{2}$. At temperatures too low for the ice to sublimate, one notes the reduced conductivity due to porosity, but as the sublimation temperature is reached, the situation changes quickly and conductivities exceeding that of compact ice are attained.

One might conclude from the location of the turnoff points that a comet where $\mathrm{H}_{2} \mathrm{O}$ is the only ice (or where other volatiles are bound as clathrates in the $\mathrm{H}_{2} \mathrm{O}$ ice) would require temperatures higher than those usually expected in order for gas-phase conductivity 
to become important. This expectation is based on models where $\mathrm{H}_{2} \mathrm{O}$ ice sublimates from the surface and thus buffers the surface temperature so that the subsurface layers are kept below the sublimation level. However, the situation becomes different when a dust mantle develops at the surface. An interesting result from the COmet SImulation (COSI) experiment (Kochan et al. 1989), illustrated in Fig. 3, is that as a dust mantle developed, the layers below the dust-ice interface did become hot enough for water vapor to be produced and to transport heat efficiently. The measured temperature profile in the ice sample took an unexpected convex shape (Fig. 3), indicating a conductivity increasing rapidly with temperature (Klinger et al. 1989). This behavior was successfully modelled by Spohn et al. (1989) using the advection by water vapor flowing down to recondense at some depth below the interface.

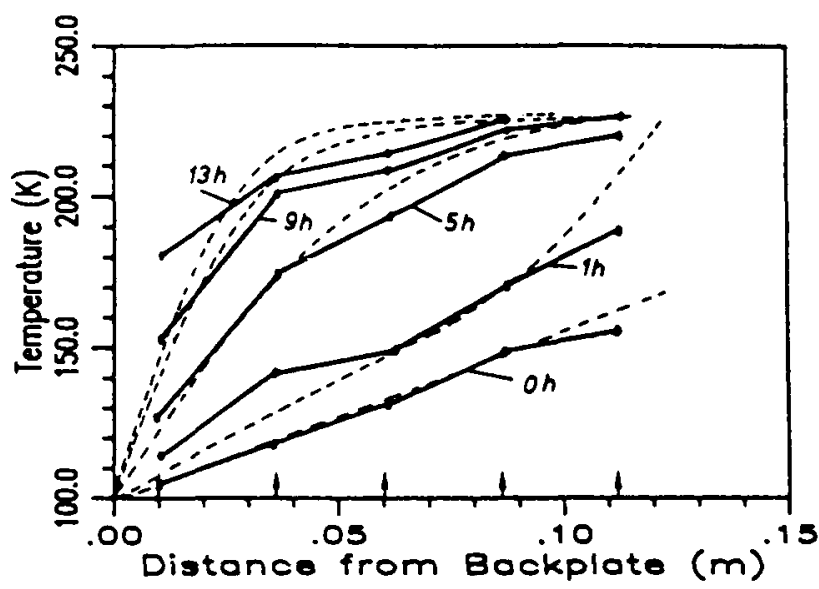

Figure 3. Temperature measurements during the COSI-1 experiment at various points in the sample material, connected by solid curves. The sublimating surface is to the right, and the backplate to the left is kept at constant temperature. Arrows mark the locations of thermocouples. The different curves are labeled by the time passed since the beginning of the experiment. Modelling results using gaseous advection are shown by dashed curves. After Spohn et al. (1989).

These results demonstrate the possible importance of gas release for controlling the thermal structure of a cometary nucleus. One should also note that gases may be released in the interior of the nucleus by processes other than the evaporation of icy constituents. It has been found by laboratory experiments (Bar-Nun et al. 1985, 1987) that amorphous ice can trap significant quantities of guest molecules, either directly upon condensation of a gas mixture or if gases flow through the ice afterward. The mechanism might be adsorption to the large specific surface area of amorphous ice, followed by trapping into closed pores as the ice mobility increases at somewhat higher temperatures (Schmitt and Klinger 1987). One may also specifically imagine microscopic hexagonal channels or closed cracks as trapping sites (Bar-Nun et al. 1988). Fig. 4 shows the trapping efficiencies for various species from a gas-rich mixture as functions of deposition temperature (Bar-Nun and Kleinfeld 1989). 
The large amounts of trapped gas at very low temperatures have been questioned (Schmitt et al. 1988a), and this is a critical issue that needs to be resolved in view of its important consequences for deducing the conditions of cometary formation. Here we only note as an example from Fig. 4 that a relative abundance of $\mathrm{CO}$ in $\mathrm{P} /$ Halley at the level of $\sim 5 \%$ (Weaver 1988) would imply a formation temperature somewhat below $50 \mathrm{~K}$ (Bar-Nun and Prialnik 1989), so in all likelihood the formation site of the material of this comet would. be trans-Neptunian. Further discussion of this argument will be given below.

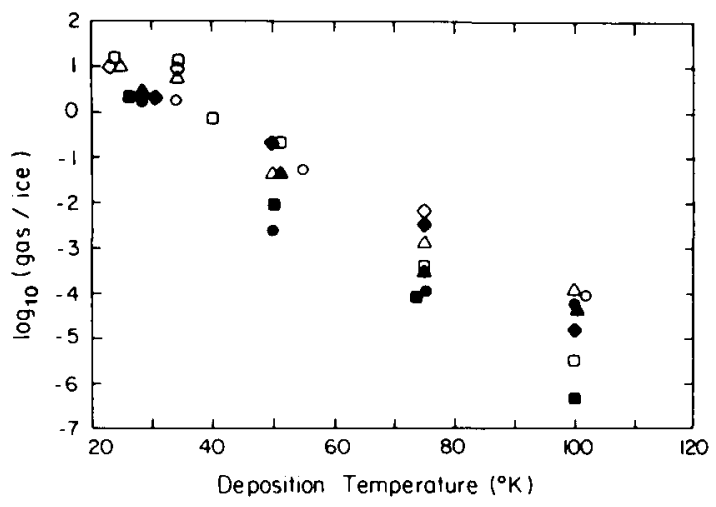

Figure 4. The ratio of the amount of trapped gas to water ice (logarithmic scale!) plotted vs. deposition temperature of the water vapor-gas mixture from experiments by Bar-Nun and coworkers. Triangles show $\mathrm{CO}$, circles show $\mathrm{N}_{2}$, diamonds show $\mathrm{CH}_{4}$, and squares show Ar. Open and filled symbols represent different gas: $\mathrm{H}_{2} \mathrm{O}$ ratios in the mixture. After Bar- $\mathrm{Nun}$ and Kleinfeld (1989).

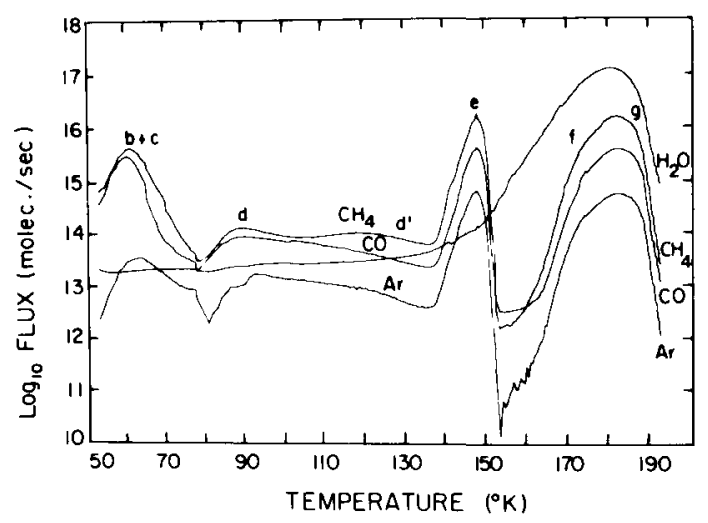

Figure 5. The fluxes of gases and water vapor produced from an amorphous low-temperature deposit upon gradual warming, as measured by Bar-Nun et al. (1988). Peaks in gas fluxes (tentatively identified by letters) represent annealing events with accompanying gas release at $\mathrm{T}$ $\$ 120 \mathrm{~K}$. The ' $e$ ' peaks come from the amorphous-cubic phase transition, and the rightmost peaks come from evaporation of the $\mathrm{H}_{2} \mathrm{O}$ ice. After Bar-Nun and Kleinfeld (1989). 
As the ice is heated, it undergoes structural modifications referred to as annealing, and gases may then be desorbed or released. Fig. 5 shows evidence from laboratory studies (Bar-Nun et al. 1988) that substantial quantities of gas could thus be produced inside a nucleus formed at a temperature $\lesssim 50 \mathrm{~K}$ in several events in the 50 to $100 \mathrm{~K}$ range. This may induce a substantial gas pressure in the porous material, and the resulting pressure gradients may lead to diffusive gas flow. Hence, heat transport by flowing gas may be important even at low temperatures. A different picture, nonetheless leading to a similar result, is illustrated in Fig. 6 (Schmitt et al. 1989). Schmitt et al. condensed an ice mixture containing large quantities of $\mathrm{CO}$ and $\mathrm{CO}_{2}$ and heated this, finding that $\sim 7 \%$ to $10 \%$ of each of these gases could remain trapped in the $\mathrm{H}_{2} \mathrm{O}$ ice after evaporation of the remainder. Obviously, then, a comet that formed including significantly more than that limit would release significant amounts of gases at low temperatures inside the nucleus. Note, however, an important difference between Figs. 5 and 6. The release of trapped gases described in Fig. 5 occurs more or less in parallel for different species, independent of their different vapor pressures, but in Fig. 6, we are dealing with the sublimation of different icy constituents, each at its characteristic temperature, reminiscent of the more classical picture of the cometary material as a mixture of individual ices (e.g., Yamamoto et al. 1983, Yamamoto 1985). Even more important is the gas release which takes place upon crystallization of the $\mathrm{H}_{2} \mathrm{O}$ ice. This is seen as a set of high peaks in the gas flux in the 140 to $150 \mathrm{~K}$ range in Fig. 5 and a drop of the $\mathrm{CO}$ and $\mathrm{CO}_{2}$ abundance ratios to essentially zero near $130 \mathrm{~K}$ in Fig. 6. The crystalline ice cannot trap gases, and clathrate formation appears, in most cases, inhibited (Schmitt et al. 1989). Therefore, as will be further discussed below, the level at which ice crystallization takes place in a cometary nucleus may be an important source of gases.
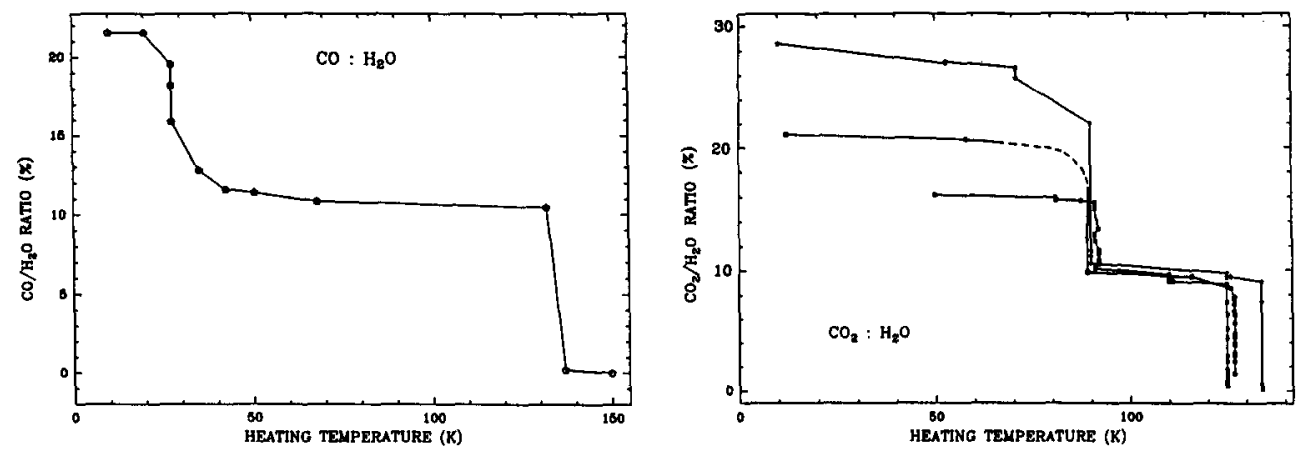

Figure 6. Ratios of $\mathrm{CO}$ (left) and $\mathrm{CO}_{2}$ (right) to $\mathrm{H}_{2} \mathrm{O}$ in an ice mixture as functions of temperature upon gradual warming in vacuum. Initially the ratios were $22 \%$ for $\mathrm{CO}$ and $16 \%, 21 \%$, and $29 \%$ for $\mathrm{CO}_{2}$. These volatiles are lost in two stages: evaporation and $\mathrm{H}_{2} \mathrm{O}$ crystallization. In between, $7 \%$ to $10 \%$ of each gas remains trapped in the amorphous $\mathrm{H}_{2} \mathrm{O}$ ice. After Schmitt et al. (1989).

Concerning the influence of low-temperature gas release and diffusion on the temperature profile, there are no experimental results as yet published. Numerical modelling 
should provide an essential tool for surveying the various possibilities as regards porosity, chemical composition and radioactive heat generation (see below), and the relevant equations are of the form:

$$
\begin{gathered}
\rho C \frac{\partial T}{\partial t}=r^{-2} \frac{\partial}{\partial r}\left\{r^{2} K \frac{\partial T}{\partial r}\right\}+Q_{h}-\rho_{g} v_{g} C_{g} \frac{\partial T}{\partial r}-p H Q_{g} \\
\frac{1}{R T} \frac{\partial P}{\partial t}=r^{-2} \frac{\partial}{\partial r}\left\{r^{2} D \frac{\partial P}{\partial r}\right\}+Q_{g}
\end{gathered}
$$

The heat diffusion equation (3) involves the bulk density $\rho$, the specific heat $C$, the gas density $\rho_{g}$, the velocity $v_{g}$ and heat capacity $C_{g}$ of the gas, the porosity $p$, the latent heat of sublimation $H$, and the gas and heat production rates per unit volume, $Q_{g}$ and $Q_{h}$. Note that $Q_{h}$ does not include the latent heat of sublimation. $H$ and $Q_{g}$ have been expressed for simplicity as referring to only one species, whereas in reality one has a sum $\sum H Q_{g}$ over many species. The two last terms are the convection and advection terms, respectively. The gas diffusion equation (4) features the total gas pressure $P$ and the diffusion coefficient $D . \quad R$ is the universal gas constant. Again, in reality the source term is a sum $\sum Q_{g}$ over many different species. The independent variables are time $t$ and distance $r$ from the center of the nucleus, assuming spherical geometry. To close the system of equations, one has $v_{g}=-D \partial P / \partial r$, and $Q_{g}$ is computed from the difference between the partial pressure $P_{g}$ and the saturation pressure $P_{\text {sat }}(T)$. $D$ can be obtained approximately using Knudsen flow (Fanale and Salvail 1984, 1987) for the case of a low gas density and using Darcy's law in the higher density continuum regime (Fanale and Salvail 1987).

Theoretical modelling using such systems of equations has as yet only been done to a limited extent. The main restriction is that all models so far have specifically dealt with the situation of a short-period comet coming close enough to the Sun to be heated from the surface. Houpis et al. (1985) made a pioneering investigation of the chemically differentiated structure and gas production rates resulting from a model accounting for subsurface sublimation of different volatiles at different depths, but they did not include the gas-solid thermal exchange at all. Fanale and Salvail (1987) included the cooling effect of $\mathrm{CO}_{2}$ flow through the $\mathrm{H}_{2} \mathrm{O}$ ice layer but not the transport of latent heat by downward flow and recondensation. Finally, Espinasse et al. (1988) and Spohn et al. (1989) have presented a full treatment of the thermal effects of gas flow, although the actual calculations by Spohn et al. were made only for pure $\mathrm{H}_{2} \mathrm{O}$ ice. Again, results are only available for surface heating. Application of such models also to internal radioactive heating would be of great interest.

\section{THE NON-VOLATILE COMPONENT}

From observations of comae and tails, dust is known to be a distinctive component of cometary material, but its mixing ratio with respect to the ice in the nucleus is not very easy to determine. The $\mathrm{P} /$ Halley exploration has brought about important results regarding both the abundance and the composition of the dust component, as discussed in detail elsewhere in this volume. For instance, the dust/ice ratio in the nucleus material 
is now estimated to be near 2 (McDonnell et al. 1990)-a value much larger than usually believed beforehand. Possible ramifications of this reevaluation have been discussed by Keller (1989). In addition, it has become clear that the silicate material usually identified with 'dust' has an important supplement in the form of organic compounds-probably complex hydrocarbons and polymers (see, e.g., Grün and Jessberger 1990). A common feature of all this material, which may be referred to as the non-volatile component, is that it has a lower vapor pressure than the dominant $\mathrm{H}_{2} \mathrm{O}$. However, the typical sublimation temperatures vary considerably, from $\sim 2000 \mathrm{~K}$ for the most refractory silicates down to $\sim 400 \mathrm{~K}$ for some organics.

What role could this component play in the thermal evolution of cometary nuclei? First of all, it is bound to be less important than that of the ice, simply because the temperature inside a comet nucleus, under normal conditions, cannot approach the level where the non-volatile material starts to sublimate. The $\mathrm{H}_{2} \mathrm{O}$ would sublimate first, either shattering the body, or leaking out and leaving behind an inert object that might start to outgas in response to heating above $400 \mathrm{~K}$. The latter scenario has some relevance to the formation of dust mantles on cometary nuclei, to be briefly discussed below.

Normally, the non-volatile component acts only by its heat capacity and thermal conductivity. In view of the importance of the organic material, whose thermal properties have not been extensively measured, even these basic parameters are not accurately known. Furthermore, the rôle of non-volatile material in heat conduction depends on the way in which it is distributed inside the nucleus. The most influential picture is that of Greenberg (1982), according to which the comet is made up of unprocessed presolar grains. These would have the silicate and organic material forming a core-mantle structure, enveloped by an ice mantle condensed in the presolar nebula. Thus one would expect the bulk conductivity to be less influenced by the non-volatile component than by the ice mantles and the contact surfaces they form with one another. However, coma grains in P/Halley seem to be very porous aggregates corresponding to large numbers of individual presolar grains (Greenberg and Hage 1990), where somehow the non-volatile material has stuck together. It is not known to what extent such structures could have formed in the deep interior of the nucleus. They could certainly be hypothesized to form by percolation processes near the surface of active regions on the nucleus. Unfortunately, the COSI results cannot yet throw much light on these questions.

\section{HEAT DIFFUSION TIME SCALE}

Obviously one cannot yet make precise and realistic statements about the thermal behavior of comets during their early stage of evolution. Theoretical modelling would certainly be helpful, but it must be recognized that there are still serious limitations to our knowledge of the values to use for the physical parameters of mixed materials. E.g., the conductivity and heat capacity of Eqs. (6) and (7) below refer to pure $\mathrm{H}_{2} \mathrm{O}$ ice, but in the actual comet we are dealing with an ice-dust mixture whose relative proportions are uncertain. This means that even very simple estimates are useful to give a rough picture of what the thermal response of the nucleus may be. Let us look at the thermal diffusivity $\kappa$. For crystalline ice at temperatures above $\sim 30 \mathrm{~K}$, this is usefully expressed by: 


$$
\kappa=\frac{K}{\rho C}
$$

and Klinger (1981) gave analytical fits for $K(T)$ and $C(T)$ based on empirical data:

$$
\begin{gathered}
K=\frac{567}{T} \quad\left(\mathrm{~W} \mathrm{~m}^{-1} \mathrm{~K}^{-1}\right) \\
C=7.49 T+90 \quad\left(\mathrm{Ws} \mathrm{kg}^{-1} \mathrm{~K}^{-1}\right)
\end{gathered}
$$

Thus the diffusivity falls off with temperature at a modest rate, and typically it is $\sim 0.1 \mathrm{~cm}^{2} / \mathrm{s}$ between 50 and $100 \mathrm{~K}$. For amorphous ice, one rather uses the proportionality $\kappa_{a} \propto v_{s} \lambda$, where $v_{s}$ is the sound speed and $\lambda$ is the mean free path of phonons. This led Klinger (1980) to an estimate of $\kappa_{a} \approx 3 \cdot 10^{-3} \mathrm{~cm}^{2} / \mathrm{s}$ and Smoluchowski (1981) to one three times larger.

The time scale for a spherical nucleus with radius $R$ to become isothermal can be estimated from a special solution to the heat diffusion equation for the center of a sphere with a constant surface temperature $T_{s}$ (Carslaw and Jaeger 1959):

$$
T_{c}=T_{s}+2 T_{s} \sum_{n=1}^{\infty}(-1)^{n} \exp \left\{-\frac{\kappa n^{2} \pi^{2} t}{R^{2}}\right\}
$$

The first term dominates after some time, and therefore the thermal relaxation time-scale can be estimated from:

$$
\tau=\frac{R^{2}}{\kappa \pi^{2}}
$$

For a nucleus with a radius of $10 \mathrm{~km}$, we obtain $\tau_{a} \sim 1 \cdot 10^{6} \mathrm{yrs}$ in the amorphous case and $\tau_{c} \sim 3 \cdot 10^{4} \mathrm{yrs}$ in the crystalline case. For a smaller nucleus, these time scales could, of course, be much shorter.

\section{Evolution Before the Active Phase}

In its early stages, a cometary nucleus is influenced by an interior heat source in the form of radioactive decay. This is expected to set up a thermal gradient such that the center becomes hottest, and as a result, some changes may be induced in the central region. In particular, gases may be released from their trapping sites and start to diffuse outward where the pressure is lower. The ices of the precometary grains probably underwent some chemical processing due to UV photolysis (Greenberg 1982) or other energetic events (e.g., Strazzulla et al. 1983). Hence there will also be some chemical energy stored in the material, and this energy is released as the temperature reaches certain critical levels where reactions start to occur. Thus the structure illustrated in Fig. 7 may develop, in which there is an 'active core' surrounded by a volatile-enriched layer where the outward-diffusing 
gases recondense. In order to predict if this will occur, one has to estimate the maximum temperature attained as a result of radioactive heating.

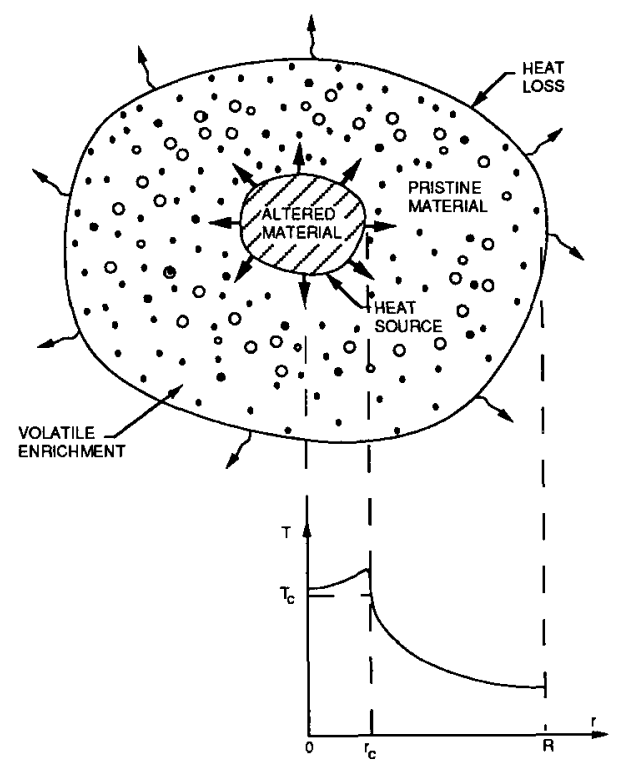

Figure 7. Tentative picture of a cometary nucleus with a central region $\left(r<r_{c}\right)$ altered by an exothermic process with critical temperature $\mathrm{T}_{c}$. Volatile migration outward from the altered core leads to recondensation into a volatile-enriched layer in the outer part of the nucleus. The diagram in the lower part indicates schematically the temperature profile to be expected.

TABLE 1. Half-lives, heat contents, and heat generation rates of different radioactive isotopes, from Prialnik (1989). $X_{d}$ is the mass fraction of dust in the cometary material.

Isotope Half-life (yrs) Heat content $(\mathrm{erg} / \mathrm{g})$

Heat generation

rate $(\mathrm{erg} / \mathrm{g} / \mathrm{s})$

$\begin{array}{llll}{ }^{40} \mathrm{~K} & 1.24 \times 10^{9} & 1.9 \times 10^{10} X_{d} & 11 X_{d} \\ { }^{232} \mathrm{Th} & 1.41 \times 10^{10} & 9.1 \times 10^{9} X_{d} & 0.45 X_{d} \\ { }^{238} \mathrm{U} & 4.47 \times 10^{9} & 4.2 \times 10^{9} X_{d} & 0.65 X_{d} \\ { }^{235} \mathrm{U} & 7.04 \times 10^{8} & 1.2 \times 10^{8} X_{d} & 1.1 X_{d} \\ { }^{26} \mathrm{Al} & 7 \times 10^{5} & <10^{11} X_{d} & <9 \times 10^{4} X_{d}\end{array}$


Table 1, from Prialnik (1989), shows rates of heat generation both for some longlived radioisotopes of relevance and for ${ }^{26} \mathrm{Al}$. The thermal relaxation time-scale estimated above is very short in comparison with the effects of the long-lived isotopes. Therefore the thermal gradient will be strongly reduced, and generally speaking, one does not expect a drastic heating from these sources (Whipple and Stefanik 1966). This was first confirmed in calculations by Lewis (1971). Later on, for instance, Yabushita and Wada (1988) found that the maximum central temperature stays very low $\left(T_{c} \lesssim 25 \mathrm{~K}\right)$, assuming a diffusivity close to that indicated above for amorphous ice. However, if a value 30 times lower is assumed (arguing, for instance, that macro-porosity might cause a drastic reduction in conductivity), then the heating becomes significant $\left(T_{c} \sim 60 \mathrm{~K}\right)$ and important modifications of the material would be expected.

The rôle of ${ }^{26} \mathrm{Al}$ is more intriguing, since this may lead to higher peak temperatures and initiate crystallization. As mentioned above, this process is exothermic and the latent energy is large enough to allow heating by as much as $80 \mathrm{~K}$ (Schmitt et al. 1988b). This is only an upper limit which might not be realistic at all, and among other effects to be discussed below, some of the heat will be conducted away. However, if crystallization occurs locally, it should be accompanied by a temperature peak. As was seen in Fig. 1, the phase transition occurs over a wide range of temperatures, but it is customary to refer to a specific crystallization temperature where all the latent heat is released at once. Recent experiments (Schmitt et al. 1988b, Bar-Nun et al. 1985, Kouchi 1987) put this temperature in the range 125 to $137 \mathrm{~K}$. From this aspect, it is natural to expect a well-defined crystallization front moving into the amorphous ice by virtue of its own latent heat. Numerical modelling of this phenomenon was first done by Herman and Podolak (1985) for the case of surface heating, but the most powerful and ingenious method was introduced by Prialnik and Bar-Nun (1987) and exploited for an investigation of interior radiogenic heating by Prialnik et al. (1987).

As illustrated in Fig. 7, crystallization ensuing from an early ${ }^{26} \mathrm{Al}$ heating would spread outward from the center of the nucleus, and thus if the heating were intense enough, the whole nucleus might be transformed into the crystalline state. Prialnik et al. (1987) found from their numerical modelling that this would occur for a nucleus with $R \sim 10 \mathrm{~km}$, if it formed with an initial ${ }^{26} \mathrm{Al}$ relative abundance of at least $\sim 10^{-8}$ (Fig. 8). Interestingly, the nucleus would then lose its trapped volatiles, as mentioned in connection with Figs. 5 and 6 . These volatiles would likely be fully outgassed, and so the significant fractions of $\mathrm{CO}$ that frequently seem to be produced from cometary nuclei give evidence that these were formed in the amorphous state and in general did not undergo such global crystallization. In terms of formation conditions, this implies a time scale longer than several million years, as shown in Fig. 8.

This result provides an interesting hint, suggesting further investigations to be pursued. In particular, it may be worthwhile to account for the thermal effects of desorption/sublimation, diffusion and readsorption/recondensation of gases. Schmitt et al. $(1988 b)$ have found that the energy expense for desorption of $7 \%$ to $10 \% \mathrm{CO}$ or $\mathrm{CO}_{2}$ during crystallization (see Fig. 6) is important: for CO, most of the latent energy would thus be consumed, and for $\mathrm{CO}_{2}$, the process would not be exothermic at all, but rather isothermal! 


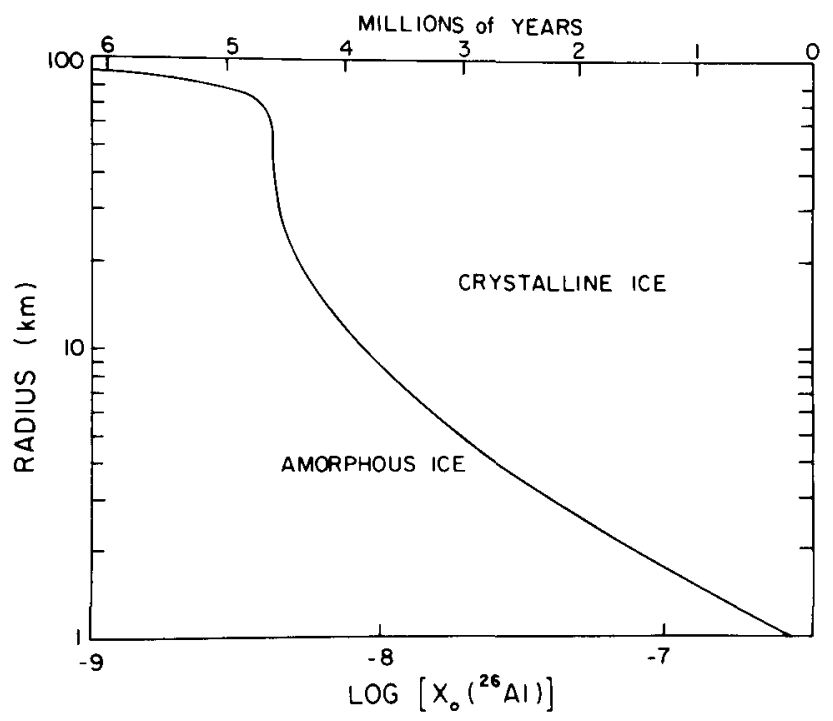

Figure 8. The maximum radius allowed (solid curve) for a spherical nucleus to remain amorphous in spite of heating by ${ }^{26} \mathrm{Al}$. The abscissa is a time scale (top of the diagram) with zero at the moment of formation of the Allende chondrite, assuming all cometary dust to incorporate ${ }^{26} \mathrm{Al}$ in abundance ratios compatible with the Allende material. At the same time (bottom of the diagram) the abscissa gives a logarithmic measure of the current ${ }^{26} \mathrm{Al}$ abundance. The later a comet is formed, the less ${ }^{26} \mathrm{Al}$ it incorporates and the larger it can be and still remain amorphous. After Prialnik et al. (1987).

It must be noted that even more drastic consequences of ${ }^{26} \mathrm{Al}$ decay than crystallization have been examined on the premise that comets might have been formed with an even larger abundance of this isotope than the $\sim 10^{-8}$ level required for crystallization. Thus Wallis (1980) found a possibility of melting the interior of the nucleus, and Irvine et al. (1980) also considered such a scenario. However, the above-mentioned more recent evidence that the basic icy constituent of cometary nuclei is porous and amorphous makes the strongly differentiated structure resulting from large-scale melting appear very unlikely. Nevertheless, it should be emphasized that the rapid decay of ${ }^{26} \mathrm{Al}$ on a time scale not too dissimilar to the one expected for comet formation leads to a delicate balance: even if neither melting nor crystallization have occurred, there is a definite possibility of structural modifications by gas release at a lower temperature and migration of volatiles toward the exterior. Therefore, upon expulsion into the Oort cloud, a typical nucleus might have a volatile-enriched layer close to the surface.

The Oort cloud phase is typically a very long-lasting one characterized by relative quiescence. The comet spends much more time than that needed for thermal relaxation under conditions of near-zero insolation. Hence, one expects a nearly isothermal nucleus with a central temperature of 20 to $30 \mathrm{~K}$ due to long-lived radionuclides and with a frozen-in 
chemical structure formed in the early phases of evolution. During the time the comets dwell in the Oort cloud, they are subject to cosmic ray bombardment, and this may drastically alter a surface layer whose thickness is estimated at several meters at most (Johnson et al. 1987). As a result, new volatile as well as non-volatile species are formed, and it is expected that this may result in peculiar behavior at the first approach to the inner Solar System, when the volatiles sublimate at relatively large distances and some of the refractories may be left behind as a surface crust, interfering with subsequent activity. However, this does not seem relevant to the large-scale thermal evolution. Nor does the surface cratering ensuing from collisional evolution in the inner Oort cloud (Stern 1988). It is of more immediate interest to consider the effects of heating by passing stars or nearby supernovae (Stern and Shull 1988).

In the latter case, one is dealing with a strong heat pulse of less than a year's duration, while a star passage in the vicinity of the comet in question will produce significant heating during hundreds of years or more. Each passing star thus affects all comets within a cylindrical tunnel through the Oort cloud, but as Weissman (1980) has shown, there is also a tunnel inside which the comets are dynamically ejected from the solar system. For very bright $O B$ stars, though, the 'heating tunnel' is much wider (Stern and Shull 1988), so they have some effect on comets later to appear in the vicinity of the Sun. The width can be estimated from the energy balance of the surface layer of an isothermal nucleus, taking the absorbed stellar radiation and re-emitted infrared radiation into account. Hence, one finds:

$$
R_{o} \sim\left\{\frac{L_{*}}{16 \pi \sigma T_{\mathrm{o}}^{4}}\right\}^{1 / 2}
$$

as the maximum distance from the star track for average surface temperatures in excess of $T_{0}$, assuming the visual and infrared Bond albedos to be equal and the nucleus to be isothermal ( $L_{*}$ is the stellar luminosity and $\sigma$ is the Stefan-Boltzmann constant). For the local subsolar point temperature, which is probably more relevant, the limiting distance is twice as large. As an example, an $O B$ star would cause subsolar point heating to $\mathrm{T}_{0}>30 \mathrm{~K}$ at distances out to 50,000 AU from the star track, while its gravitational perturbations would expel the comets from the Oort cloud out to, typically, one-tenth of this distance.

However, it is important to estimate how deeply the heating will penetrate. The heat pulse is attenuated exponentially with depth, and the relevant scale height (the thermal skin depth) is given by:

$$
S=\sqrt{\frac{\kappa \tau}{\pi}}
$$

where $\tau$ is the duration of the pulse. With a standard amorphous ice diffusivity $\kappa_{a}$ (see above), one obtains $S=17 \mathrm{~m}$ for a heating period of 100 yrs. Fig. 9 shows results by Stern and Shull for the fraction of Oort cloud comets heated to different surface temperatures. Apparently heating events reaching $\sim 30 \mathrm{~K}$, where some noteworthy alteration of the material might occur, are experienced by $\sim 10 \%$ of all comets. Since these heating events are dominated by OB stars, a typical duration would be $\tau \leqslant 10^{4}$ yrs, which implies $S \leqslant 100 \mathrm{~m}$. 
Hence, some alteration occurs in at least a significant fraction of the comets, but it is restricted to a surface layer of $\leqslant 100 \mathrm{~m}$ depth. Nearby supernovae give somewhat more intense heating $\left(T_{o} \sim 50 \mathrm{~K}\right)$ to all comets, but this surface layer is restricted to $\leq 0.5 \mathrm{~m}$ (Stern and Shull 1988).

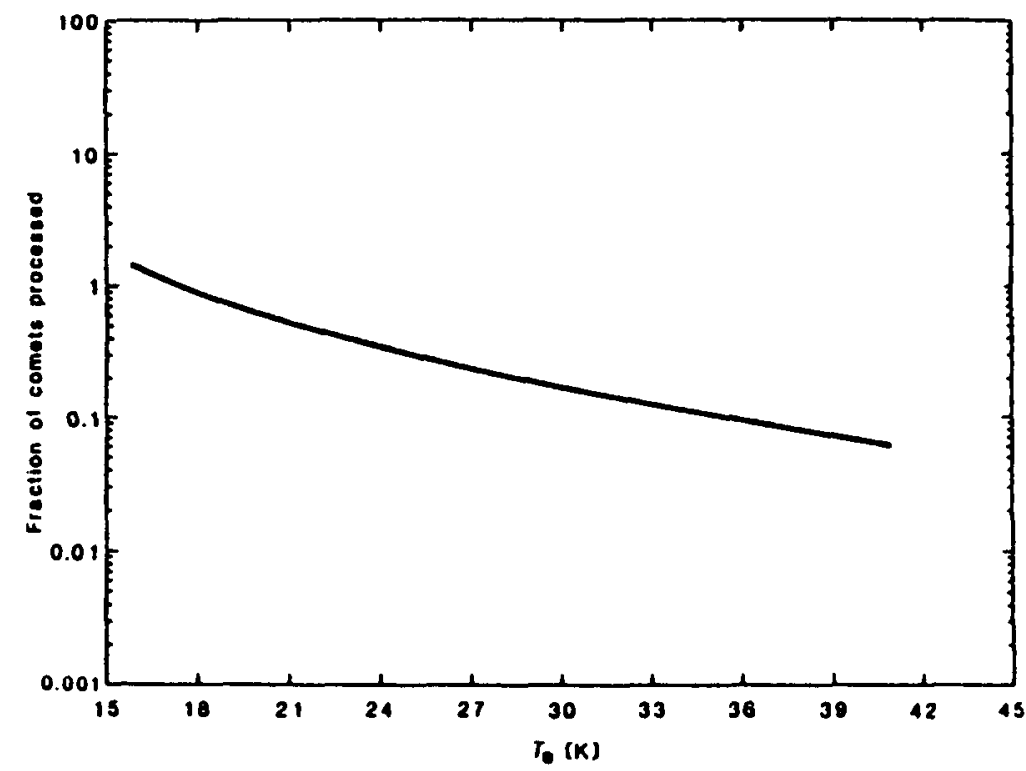

Figure 9. The fraction of comets heated to equilibrium surface temperature $\mathrm{T}_{\boldsymbol{e}}$ or more as a result of encounters with all types of stars during $4.5 \mathrm{Gyr}$. Notice that heating to more than $30 \mathrm{~K}$ is not a very unlikely outcome. After Stern and Shull (1988).

\section{Evolution in the Active Cometary Phase}

The loss of orbital angular momentum and subsequent entry into the planetary system marks the beginning of the final stage of cometary evolution. Let us concentrate on the thermal effects experienced by active comets, which, at perihelion, pass close enough to the Sun for $\mathrm{H}_{2} \mathrm{O}$ ice to sublimate from the surface of the nucleus. The total time span of the evolution caused by these effects is typically $\$ 10^{6} \mathrm{yrs}$, and the driving mechanism is a large number of heating events as the comet returns to successive perihelia. If we consider typical short-period comets, this number is, in general, $z 10^{3}$. The events often occur quasi-periodically during intervals of orbital stability, but on the whole the sequence is rather irregular. Perturbations of the perihelion distance and orbital period (see Carusi et al. 1985) lead to variations of the amplitude and frequency of solar heating events.

We are now considering heating of the nucleus from the outside to maximum temperatures well in excess of the crystallization temperature. The ice will therefore start to 
crystallize from the surface, and at any particular instant, there will be a surface layer of crystalline ice on top of an amorphous interior. This means quite an important chemical differentiation expected of all comets having passed close to the Sun at least once. Physically, we are dealing with the situation depicted in Fig. 10, at least in an initial stage. Deep enough, the ice remains quasi-pristine, amorphous and gas-rich. But temperatures increase toward the surface when the comet is close to the Sun, and levels are reached where trapped gas is released or individual volatile constituents sublimate (cf. Figs. 5 and 6). Part of this gas recondenses slightly below the point of release into a volatile-enriched layer, but part of it diffuses upward through warmer material. Hence, there is a diffusive flux of gas approaching the surface. According to Schmitt et al. (1989), $7 \%$ to $10 \%$ of the gas remains trapped in the warmer amorphous ice, but at the level of crystallization, this is released as well. Therefore, at this level, one has an additional source of gas, and there may be a significant flux of gases passing through the crystalline crust and produced interior to it. The essentials of the picture just outlined are not changed if the gas trapping results of Bar-Nun et al. (1988) are considered rather than those of Schmitt et al.

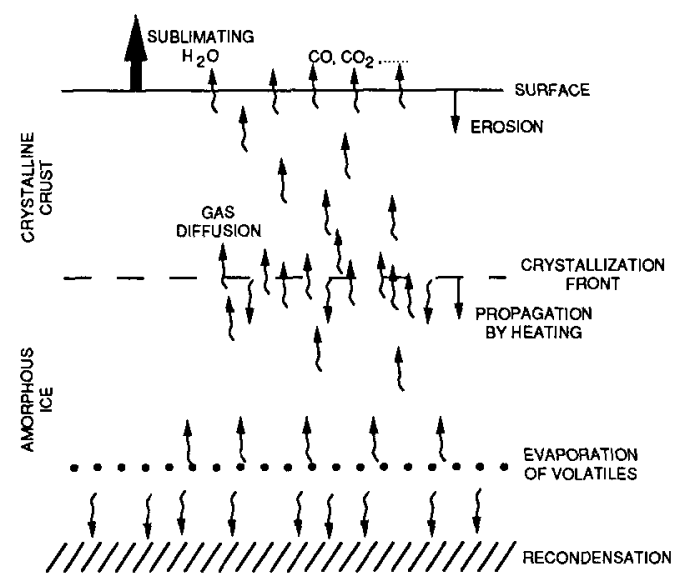

Figure 10. Sketch of a possible surface structure in a short-period comet nucleus including $\mathrm{H}_{2} \mathrm{O}$ crystallization with accompanying gas release, and evaporation of additional volatiles at interior levels.

It should be noted that this picture remains qualitative and may be in need of substantial modification as more knowledge is gathered. Nevertheless, it is clear that as time passes, the surface is eroded due to $\mathrm{H}_{2} \mathrm{O}$ sublimation and consequently, the whole structure is bound to move down through the nuclear material. If there were a steady state, the structure would be rigid, and, according to the present picture, volatiles would always be produced at the surface in the same proportions as they exist in the pristine material. Under that assumption, one would thus be justified in interpreting the relative production rates of volatiles in comet $\mathrm{P} / \mathrm{Halley}$ in terms of the bulk composition of the nucleus. However, in the course of an apparition, one might well see a variation of, for 
example, the thickness of the crystalline crust, and hence it is not certain that the observed production rates at any given instant should match the interior composition.

Indications about this may be obtained from thermal modelling of chemical differentiation as discussed above. At present, results have been found by Prialnik and Bar-Nun $(1987,1988)$, who treat the thermal effects of crystallization considering that all the latent heat is available as a source term in the heat diffusion. In addition, Espinasse et al. (1988) treated the full problem of heat and gas diffusion assuming that all the latent heat of crystallization is consumed by desorbing trapped gases. The latter investigation led to the result that the crystallization front can remain very close to the surface $(\lesssim 1 \mathrm{~m})$, in agreement with earlier suggestions based on model calculations where the thermal effect of crystallization was disregarded (Rickman and Froeschlé 1983, Kührt 1984). However, like Herman and Podolak (1985), Prialnik and Bar-Nun $(1987,1988)$ included this effect so that the front was propelled downward by its own latent heat. As a result, it would stop only at depths $\gtrsim 10 \mathrm{~m}$. Further work is needed in order to clarify which result is most relevant for real comets. Fig. 11 shows the outcome of a model for comet P/Halley assuming pure, porous ice (Prialnik and Bar-Nun 1987), where we see crystallization proceeding in several spurts reaching further and further down into the nucleus. After several hundred revolutions, the whole nucleus would probably crystallize. Each new spurt is initiated when the hot surface comes closer than $\sim 15 \mathrm{~m}$, and it stops when the crystallization front reaches layers that are too cold to be activated. This occurs successively at larger and larger depths due to the progressive heating of the whole amorphous interior.

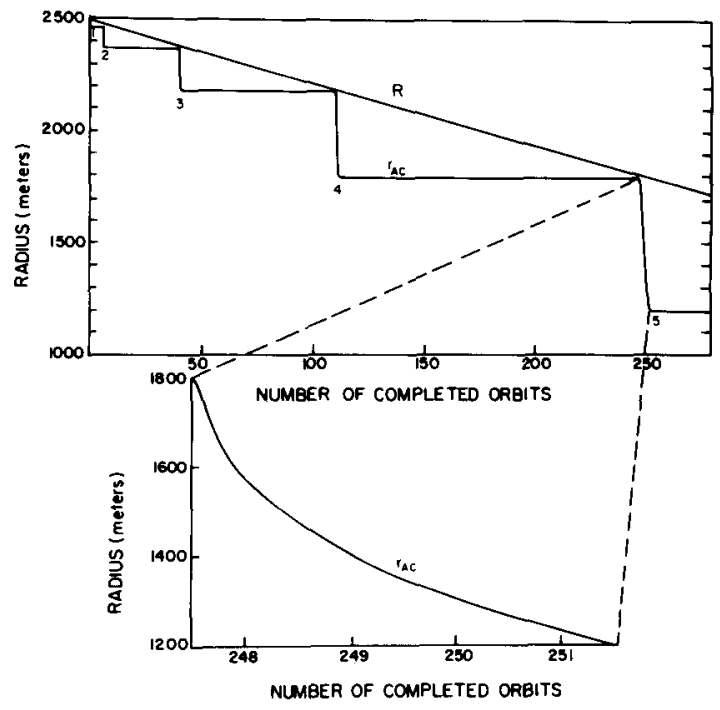

Figure 11. Evolution of the radius $\mathrm{R}$ of the nucleus and the radius $\mathrm{r}_{A C}$ of the amorphous core for a comet moving in the orbit of $\mathrm{P} / \mathrm{Halley}$. Initially the nucleus is completely amorphous with a radius of $2.5 \mathrm{~km}$. The lower diagram shows a blowup of the variation of $\mathrm{x}_{A C}$ during the fifth crystallization round. After Prialnik and Bar-Nun (1987). 
However, if the same model is run for a dusty ice nucleus (Prialnik and Bar-Nun 1988), the latent heat released per gram of ice must also heat a comparable amount of dust, and as a general result, the front then tends to remain at a more constant depth. Dust mantle formation and ice porosity are also influential parameters, so one cannot predict the details of the ensuing evolution with high confidence. In any case, it does not appear likely that the $\mathrm{P} /$ Halley nucleus would have crystallized entirely, even assuming a large number of past revolutions as suggested by McIntosh and Hajduk (1983) and Hughes (1985).

Finally, it is of obvious interest to know how the secular evolution of deep interior temperatures varies between different kinds of cometary orbits. For instance, taking a short-period comet of the Jupiter family such as those currently envisaged as targets for space missions (Neugebauer 1987, Wood 1987), will such a comet have any quasi-pristine interior left, and if so, at what depth? There is not yet any full answer to this question (see Weissman and Stern 1990). The estimated active lifetimes (i.e., lifetimes as observable comets) of $\sim 10^{4}$ yrs (Kresák 1985, Fernández 1985) are far shorter than the thermal relaxation time-scale for amorphous ice as estimated above. There would hardly be time enough to completely relax even a crystalline nucleus, and moreover, the orbit of the comet and thus the conditions to which the nucleus would relax usually change on a still much shorter time-scale (see Carusi et al. 1985).

The interior thermal structure of a typical Jupiter family comet may therefore be very complicated and unpredictable, reflecting in part the chaotic nature of orbital evolution. Nevertheless, as a first attempt, one would naturally try to estimate an equilibrium internal temperature as a function of the orbital parameters. This was first done by Klinger $(1981,1983)$, who took an energy balance condition of the usual type:

$$
\frac{1}{4}\left(1-A_{v}\right) F_{\odot} r_{\odot}^{-2}=\varepsilon \sigma T_{s}^{4}+H\left(T_{s}\right) Z\left(T_{s}\right)-\left.K \frac{\partial T}{\partial z}\right|_{s}
$$

for an isothermal surface. Here $A_{v}$ is the visual Bond albedo and $\varepsilon$ is the thermal emissivity. $F_{\odot}$ is the solar constant, and $r_{\odot}$ is heliocentric distance, so the left-hand member represents the absorbed flux of solar energy, and the first term in the right-hand member gives the heat loss due to thermal radiation. In the second term, representing the energy expense for $\mathrm{H}_{2} \mathrm{O}$ sublimation, $H$ is the latent heat and $Z$ is the gas flux leaving the nucleus. The third term is the heat flux conducted downward into the nucleus ( $z$ is the depth below the surface). Klinger argued that an average surface temperature $T_{m}$ could be derived, assuming that the average heat flow around the whole orbit vanishes, yielding:

$$
\frac{F_{\odot}\left(1-A_{v}\right)}{4 a^{2} \sqrt{1-e^{2}}}=\varepsilon \sigma T_{m}^{4}+\langle H Z\rangle
$$

where $a$ is the semimajor axis and $e$ the eccentricity of the orbit. In equilibrium, at infinite depth, the constant temperature should equal this average, called the orbital mean temperature. One is thus in essence estimating the average as:

$$
T_{m}=\left\langle T_{s}^{4}\right\rangle^{1 / 4}
$$


Kührt (1984) correctly argued that a straightforward time average of the surface temperature, $T_{c}=\left\langle T_{s}\right\rangle$, is physically more meaningful, and McKay et al. (1986) pointed out that the temperature dependence of heat conductivity must be taken into account since the interior couples more or less strongly to the surface depending on the temperature. Using the anti-derivative $\widehat{K}(T)$ of the conductivity function $K(T)$, i.e., $d \widehat{K}(T) / d T \equiv K(T)$, they devised an analytical formula:

$$
T_{d}=\widehat{K}^{-1}\left\{\left\langle\widehat{K}\left(T_{s}\right)\right\rangle\right\}
$$

for the equilibrium temperature $T_{d}$. Furthermore, they used an idealized model neglecting sublimation losses as well as conduction at the surface to compute the time average in Eq. (15) and to point out the discrepancies with Klinger's results.
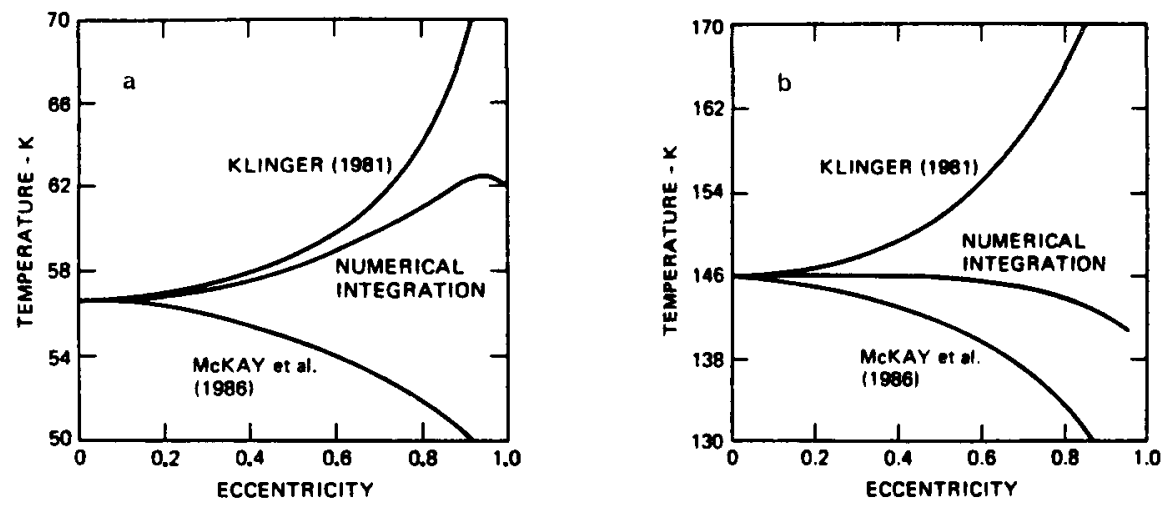

Figure 12. The equilibrium central temperature of a cometary nucleus as a function of orbital eccentricity is shown for two values of the semimajor axis: (a) $a=20 \mathrm{AU}$, representing a Halleytype comet; (b) $a=3 \mathrm{AU}$, representing a Jupiter-family comet. Idealized models by Klinger (1981) and McKay et al. (1986) are compared with the results of numerical integrations of a thermal model. After Herman and Weissman (1987).

Herman and Weissman (1987) finally carried out numerical integrations in a more realistic model which included both sublimation and heat flow. They confirmed the validity of the McKay et al. formula, but their model, of course, gave different results, and Fig. 12 shows the comparison with the earlier idealized models. One might have the awkward impression that we do not know very much about the true equilibrium temperatures, but basically we see the effects of including or excluding the sublimation and conduction terms in Eq. (12). There is no question that the 'numerical integration' curve is the relevant one from this aspect. Among the premises upon which it rests, we find that an icy surface in active sublimation is assumed, and that the cooling effect of surface erosion (Rickman and Fernández 1986) is neglected.

The formation of dust mantles (see below) will influence the near-surface temperatures drastically when the comet is close to the Sun, but estimating the effect on the interior 
temperature by cutting off sublimation in the surface energy budget, Herman and Weissman (1987) found this to be of little importance. Table 2, from Herman and Weissman, shows typical results for short-period comets, and it is interesting to note that the internal temperatures are generally above the crystallization limit. One would therefore expect these nuclei to crystallize completely in due time. However, one has to keep in mind that this time is very long compared with the expected active lifetime. For an active comet, one might expect a layered temperature structure resulting from the different orbits that the comet has had in the past (Weissman 1986a), but even this expectation appears doubtful in the light of recent calculations by Bar-Nun et al. (1989), who studied the evolution of comet $\mathrm{P} /$ Tempel 1. They found that even after more than 100 revolutions, the thermal structure of most of the nucleus - the amorphous interior - had not changed perceptibly. The crystalline crust stayed relatively thin, although thicker than expected sampling depths, and any thermal layering would apparently be confined to the outer regions of that crust. Extension of such calculations to time scales $\sim 10^{3}$ revolutions, comparable to expected cometary lifetimes, would be of great interest.

TABLE 2. Orbital elements and central temperatures for different shortperiod comets, from Herman and Weissman (1987). $T_{c}$ is the temperature with a free-sublimating icy surface, and $T_{c}^{\prime}$ with a mantle-covered surface choking the sublimation.

\begin{tabular}{lcccc}
\hline Comet & $\begin{array}{c}\text { Perihelion } \\
\text { distance }(\mathrm{AU})\end{array}$ & $\begin{array}{c}\text { Orbital } \\
\text { period (yrs) }\end{array}$ & $T_{c}(\mathrm{~K})$ & $T_{c}^{\prime}(\mathrm{K})$ \\
\hline $\begin{array}{l}\text { Encke } \\
\begin{array}{l}\text { Honda-Mrkos- } \\
\text { Pajduśáková }\end{array}\end{array}$ & 0.541 & 3.30 & 171.0 & 183.4 \\
$\begin{array}{l}\text { Tempel 2 } \\
\text { Wild 2 }\end{array}$ & 1.381 & 5.28 & 149.8 & 154.9 \\
d'Arrest & 1.491 & 5.29 & 150.8 & 156.9 \\
Kopff & 1.291 & 6.17 & 144.7 & 148.9 \\
Churyumov- & 1.572 & 6.38 & 141.9 & 146.1 \\
Gerasimenko & 1.298 & 6.43 & 143.4 & 147.0 \\
Halley & 0.587 & 6.59 & 140.8 & 145.2 \\
\hline
\end{tabular}

Comets in general, and short-period ones in particular, appear to experience a distinctive evolution by dust mantling of the nucleus surface (for a recent review, see Rickman 
and Huebner 1990). This is an essential part of the near-surface chemical differentiation expected to appear during the active cometary phase. The ideas about the formation of dust mantles employed in models have as yet remained rather preliminary. Mostly, they are based on considering the dust component of cometary nuclei as a collection of grains with a wide size spectrum, in agreement with the grains observed in comae and tails. Grains that are too large to be entrained by the outflowing gas (Whipple 1950) reside on the nucleus, and a coherent layer may form as a result of grain trapping (Shul'man 1972) as the surface coverage grows large enough. Some models which include a diffusive gas flow through this layer (e.g., Rickman et al. 1990) predict a nearly complete choking of the gas flow for a mantle thickness as small as $\sim 1 \mathrm{~cm}-$ a phenomenon occurring on a short time-scale under a wide range of conditions. Needless to say, such mantles would be very unstable and prone to rupture or blowoff in response to changes of the orbit or spin of the comet.

However, in the light of recent findings, in particular, the discovery of the organic component of cometary dust and the identification of dust grains as porous aggregates, the models now appear to be based on oversimplified concepts. Thus the time should be ripe for developing a new generation of models, taking account of these features as well as the wealth of experimental results (COSI and others). Probably the polymers, forming part of the organic material, play an important rôle for grain sticking and buildup of aggregates. Dust mantles involving such material may have a rather consolidated structure, rather than being loose collections of grains. Surface temperatures on top of the mantles may reach well above $400 \mathrm{~K}$ for comets with perihelia well inside the Earth's orbit - for an observational indication regarding $\mathrm{P} /$ Halley, see Lamarre (1989). Evaporation of organic species may then cause surface erosion and structural modifications of the dust mantles.

What changes of the physical structure can we expect in the surface layers of the nucleus as a result of the thermal and chemical evolution? On the basis of the mechanical properties expected of cometary material, it appears from the estimates by Tauber and Kührt (1987), Kührt et al. (1986), and Green (1986) that cracking should occur as a result of the steep temperature gradients. The idea of subsurface volatile pockets recently got new impetus from the experimental results of Bar-Nun et al. (1987) and Laufer et al. (1987), indicating that the gases released upon crystallization cannot escape freely through the crystalline crust, but accumulate by dynamic percolation processes in pockets that explode as a result of the buildup of large enough gas pressure. Prialnik and Bar-Nun (1988) stress this mechanism as a likely explanation of eruptive behavior in, for instance, comet $\mathrm{P} / \mathrm{Halley}$. Again, we are dealing with an interesting suggestion showing the preliminary nature of the picture outlined in Fig. 10. More work is clearly needed, including also thermal cracking and meteoritic gardening (Fernández 1990) to find out how gases are transported through the crystalline crust and the dust mantle developing on top of it.

\section{Concluding Remarks}

The thermal behavior of porous, initially amorphous and gas-rich material, perhaps in the form of a mixture with different ices, moving in cometary orbits is a field rich in possibilities where many interesting consequences still remain to be explored. Moreover, it 
can be foreseen that continued laboratory work, as well as future space missions of rendezvous or sample-return type, will bring even more new aspects into the picture. Modelling work needs to be pursued further in order to meet these challenges. A final remark, awaiting such new developments, is that the present state of research seems more virginal than the cometary nuclei themselves (see Weissman 1986a).

\section{Acknowledgements}

This work was supported by grants nr. F-FU 3271-301 and R-RA 3271-304 from the Swedish Natural Science Research Council. Discussions with J. Klinger, B. Schmitt, and A. Bar-Nun have been highly informative and stimulating and are greatly appreciated.

\section{References}

Bar-Nun, A., and Kleinfeld, I. (1989) 'On the Temperature and Gas Composition in the Region of Comet Formation,' Icarus 80, 243-253.

Bar-Nun, A., and Prialnik, D. (1989) 'Formation of Comets - Laboratory Studies and Modelling,' invited paper at Asteroids, Comets, Meteors III, Uppsala Univ.

Bar-Nun, A., Herman, G., Laufer, D., and Rappaport, M.L. (1985) 'Trapping and Release of Gases by Water Ice and Implications for Icy Bodies,' Icarus 63, 317-332.

Bar-Nun, A., Dror, J., Kochavi, E., and Laufer, D. (1987) 'Amorphous Water Ice and Its Ability to Trap Gases,' Phys. Rev. B 35, 2427-2435.

Bar-Nun, A., Kleinfeld, I., and Kochavi, E. (1988) 'Trapping of Gas-Mixtures in Amorphous Water Ice,'Phys. Rev. B 38, 7749-7754.

Bar-Nun, A., Heifetz, E., and Prialnik, D. (1989) 'Thermal Evolution of Comet P/Tempel 1 - Representing the Group of Targets for the CRAF and CNSR Missions,' Icarus 79, 116-124.

Cameron, A.G.W. (1985) 'Formation and Evolution of the Primitive Solar Nebula,' in D.C. Black and M.S. Matthews (eds.), Protostars and Planets II, Univ. Arizona Press, Tucson, pp. 1073-1099.

Carslaw, H.S., and Jaeger, J.C. (1959) Conduction of Heat in Solids, Oxford Univ. Press, London.

Carusi, A., Kresák, L., Perozzi, E., and Valsecchi, G.B. (1985) Long-Term Evolution of Short-Period Comets, Adam Hilger, Bristol.

Daniels, P.A., and Hughes, D.W. (1981) 'The Accretion of Cosmic Dust - A Computer Simulation,' Mon. Not. R. Astron. Soc. 195, 1001-1009.

Donn, B., and Hughes, D.W. (1985) 'On the Structure of the Cometary Nucleus,' Bull. Amer. Astr. Soc, 17, 689 (abstract).

Donn, B., and Rahe, J. (1982) 'Structure and Origin of Cometary Nuclei,' in L.L. Wilkening (ed.), Comets, Univ. Arizona Press, Tucson, pp. 203-226. 
Espinasse, S. (1989) 'Modelisation du comportement thermique et de la différenciation chimique des noyaux de comètes,' Doctoral thesis, Univ. of Grenoble.

Espinasse, S., Schmitt, B., and Klinger, J. (1988) 'Modelisation du comportement thermique et la différenciation chimique des noyaux de comètes: Résultats préliminaires,' in M.C. Festou and D. Chabod (eds.), Comptes Rendus des Journées de Planétologie (CNRS-INSU), Observatoire de Besançon, pp. 207-210.

Fanale, F.P., and Salvail, J.R. (1984) 'An Idealized Short-Period Comet Model: Surface Insolation, $\mathrm{H}_{2} \mathrm{O}$ Flux, Dust Flux, and Mantle Evolution,' Icarus 60, 476-511.

Fanale, F.P., and Salvail, J.R. (1987) 'The Loss and Depth of $\mathrm{CO}_{2}$ Ice in Comet Nuclei,' Icarus 72, 535-554.

Fernández, J.A. (1985) 'Dynamical Capture and Physical Decay of Short-Period Comets,' Icarus 64, 308-319.

Fernández, J.A. (1990) 'Collisions of Comets with Meteoroids,' in C.-I. Lagerkvist, H. Rickman, B.A. Lindblad, and M. Lindgren (eds.), Asteroids, Comets, Meteors III, Uppsala Univ., pp. 309-312.

Fernández, J.A., and Ip, W.-H. (1981) 'Dynamical Evolution of a Cometary Swarm in the Outer Planetary Region,' Icarus 47, 470-479.

Geiss, J. (1987) 'Composition Measurements and the History of Cometary Matter,'Astron. Astrophys. 187, 859-866.

Ghormley, J.A. (1968) 'Enthalpy Changes and Heat Capacity Changes in the Transformation from High Surface Area Amorphous Ice to Stable Hexagonal Ice,' J. Chem. Phys. 48, 503-508.

Green, J.R. (1986) 'Stress, Fracture, and Outburst in Cometary Nuclei,' Bull. Amer. Astr. Soc. 18, 800 (abstract).

Greenberg, J.M. (1982) 'What Are Comets Made Of? A Model Based on Interstellar Dust,' in L.L. Wilkening (ed.), Comets, Univ. Arizona Press, Tucson, pp. 131-163.

Greenberg, J.M. (1983) 'Interstellar Dust, Comets, Comet Dust and Carbonaceous Meteorites,' in C.-I. Lagerkvist and H. Rickman (eds.), Asteroids, Comets, Meteors, Uppsala Univ., pp. 259-268.

Greenberg, J.M. (1986) 'Fluffy Comets,' in C.-I. Lagerkvist, B.A. Lindblad, H. Lundstedt, and H. Rickman (eds.), Asteroids, Comets, Meteors II, Uppsala Univ., pp. 221-223.

Greenberg, J.M., and Grim, R. (1986) 'The Origin and Evolution of Comet Nuclei and Comet Halley Results,' in B. Battrick, R. Rolfe, and R. Reinhard (eds.), Exploration of Halley's Comet, ESA SP-250, vol. II, pp. 255-263.

Greenberg, J.M., and Hage, J.I. (1990) 'From Interstellar Dust to Comets: A Unification of Observational Constraints,' Astrophys. J., in press.

Grün, E., and Jessberger, E.K. (1990) 'Dust,' in W.F. Huebner (ed.), Physics and Chemistry of Comets, Springer Verlag, in press.

Herman, G., and Podolak, M. (1985) 'Numerical Simulation of Comet Nuclei, I. Water-Ice Comets,' Icarus 61, 252-266. 
Herman, G., and Weissman, P.R. (1987) 'Numerical Simulation of Cometary Nuclei. III. Internal Temperatures of Cometary Nuclei,' Icarus 69, 314-328.

Houpis, H.L.F. (1990) 'Models of Cometary Nuclei,' in J. Mason and P. Moore (eds.), Comet Halley 1986: Worldwide Investigations, Results and Interpretations, EllisHorwood Publ. Co., in press.

Houpis, H.L.F., Ip, W.-H., and Mendis, D.A. (1985) 'The Chemical Differentiation of the Cometary Nucleus: The Process and Its Consequences,' Astrophys. J. 295, 654-667.

Hughes, D.W. (1985) 'The Size, Mass, Mass Loss and Age of Halley's Comet,' Mon. Not. R. Astr. Soc. 213, 103-109.

Irvine, W.M., Leschine, S.B., and Schloerb, F.P. (1980) 'Thermal History, Chemical Composition and Relationship of Comets to the Origin of Life,' Nature 283, 748-749.

Johnson, R.E., Cooper, J.F., Lanzerotti, L.J., and Strazzulla, G. (1987) 'Radiation Formation of a Non-Volatile Comet Crust,'Astron. Astrophys. 187, 889-892.

Keller, H.U. (1989) 'Comets - Dirty Snowballs Or Icy Dirtballs?,' in J. Hunt and T.D. Guyenne (eds.), Physics and Mechanics of Cometary Materials, ESA SP-302, pp. 39-45.

Klinger, J. (1980) 'Influence of a Phase Transition of Ice on the Heat and Mass Balance of Comets,' Science 209, 271-272.

Klinger, J. (1981) 'Some Consequences of a Phase Transition of Water Ice on the Heat Balance of Comet Nuclei,' Icarus 47, 320-324.

Klinger, J. (1983) 'Classification of Cometary Orbits Based on the Concept of Orbital Mean Temperature,' Icarus 55, 169-176.

Klinger, J., Benkhoff, J., Espinasse, S., Grün, E., Ip, W.-H., Joó, F., Keller, H.U., Kochan, H., Kohl, H., Roessler, K., Seboldt, W., Spohn, T., and Thiel, K. (1989) 'How Far Do Results of Recent Simulation Experiments Fit Current Models of Cometary Nuclei?,' Proc. 19th Lunar Planet. Sci. Conf., Lunar and Planetary Inst., Houston, pp. 493-497.

Kochan, H., Feuerbacher, B., Joó, F., Klinger, J., Seboldt, W., Bischoff, A., Düren, H., Stöffler, D., Spohn, T., Fechtig, H., Grün, E., Kohl, H., Krankowsky, D., Roessler, K., Thiel, K., Schwehm, G., and Weishaupt, U. (1989) 'Comet Simulation Experiments in the DFVLR Space Simulators,' Adv. Space Res. 9, No. 3, pp. 113-122.

Kouchi, A. (1987) 'Vapour Pressure of Amorphous $\mathrm{H}_{2} \mathrm{O}$ Ice and Its Astrophysical Implications,' Nature 330, 550-552.

Kresák, L. (1985) 'The Aging and Lifetimes of Comets,' in A. Carusi and G.B. Valsecchi (eds.), Dynamics of Comets: Their Origin and Evolution, D. Reidel Publ. Co., Dordrecht/Boston/Lancaster, pp. 279-302.

Kührt, E. (1984) 'Temperature Profiles and Thermal Stresses in Cometary Nuclei,' Icarus 60, $512-521$.

Kührt, E., Möhlmann, D., Giese, B., and Tauber, F. (1986) 'Thermal Stresses and Dust Dynamics on Comets,' in B. Battrick, R. Rolfe, and R. Reinhard (eds.), Exploration of Halley's Comet, ESA SP-250, vol. II, pp. 385-388. 
Lamarre, J.-M. (1989) 'Détection de l'émission infra-rouge du noyau de la comète de Halley à l'aide de l'instrument IKS embarqué sur les sondes spatiales Vega,' Doctoral thesis, Univ. of Paris VII.

Laufer, D., Kochavi, E., and Bar-Nun, A. (1987) 'Structure and Dynamics of Amorphous Water Ice,' Phys. Rev. B 36, 9219-9227.

Lewis, J.S. (1971) 'Satellites of the Outer Planets: Their Physical and Chemical Nature,' Icarus 15, 174-185.

Lewis, J.S., and Prinn, R.G. (1980) 'Kinetic Inhibition of $\mathrm{CO}$ and $\mathrm{N}_{2}$ Reduction in the Solar Nebula,' Astrophys. J. 238, 357-364.

McDonnell, J.A.M., Pankiewicz, G.S., Birchley, P.N.W., Green, S.F., and Perry, C.H. (1990) 'Dust Mass Distributions: The Perspective from Giotto's Measurements at P/Halley,' in Proc. of the workshop on Analysis of Returned Comet Nucleus Samples, Milpitas, $\mathrm{CA}$, in press.

McIntosh, B.A., and Hajduk, A. (1983) 'Comet Halley Meteor Stream: A New Model,' Mon. Not. R. Astr. Soc. 205, 931-943.

McKay, C.P., Squyres, S.W., and Reynolds, R.T. (1986) 'Methods for Computing Comet Core Temperatures,' Icarus 66, 625-629.

Morfill, G.E., Tscharnuter, W., and Völk, H.J. (1985) 'Dynamical and Chemical Evolution of the Protoplanetary Nebula,' in D.C. Black and M.S. Matthews (eds.), Protostars and Planets II, Univ. Arizona Press, Tucson, pp. 493-533.

Neugebauer, M. (1987) 'The Comet Rendezvous Asteroid Flyby Mission,' in E.J. Rolfe and B. Battrick (eds.), Diversity and Similarity of Comets, ESA SP-278, pp. 517-522.

Patashnick, H., Rupprecht, G., and Schuerman, D.W. (1974) 'Energy Source for Comet Outbursts,' Nature 250, 313-314.

Peale, S.J. (1989) 'On the Density of Halley's Comet,' Icarus 82, 36-49.

Prialnik, D. (1989) 'Thermal Evolution of Cometary Nuclei,' Adv. Space Res. 9, No. 3, pp. 25-40.

Prialnik, D., and Bar-Nun, A. (1987) 'On the Evolution and Activity of Cometary Nuclei,' Astrophys. J. 313, 893-905.

Prialnik, D., and Bar-Nun, A. (1988) 'The Formation of a Permanent Dust Mantle and Its Effect on Cometary Activity,'Icarus 74, 272-283.

Prialnik, D., Bar-Nun, A., and Podolak, M. (1987) 'Radiogenic Heating of Comets by ${ }^{26} \mathrm{Al}$ and Implications for Their Time of Formation,' Astrophys. J. 319, 993-1002.

Rickman, H. (1986) 'Masses and Densities of Comets Halley and Kopff', in O. Melita (ed.), Comet Nucleus Sample Return, ESA SP-249, pp. 195-205.

Rickman, H. (1987) 'Physical Evolution of Comets,' in Z. Ceplecha and P. Pecina (eds.), Interplanetary Matter, Publ. Astron. Inst. Czech. Acad. Sci. No. 67, vol. 2, pp. 37-46.

Rickman, H. (1989) 'The Nucleus of Comet Halley: Surface Structure, Mean Density, Gas and Dust Production,' Adv. Space Res. 9, No. 3, pp. 59-71. 
Rickman, H., and Fernández, J.A. (1986) 'Formation and Blowoff of a Cometary Dust Mantle,' in O. Melita (ed.), Comet Nucleus Sample Return, ESA SP-249, pp. 185-194. Rickman, H., and Froeschlé, C. (1983) 'Thermal Models for the Nucleus of Comet P/ Halley,' in T.I. Gombosi (ed.), Cometary Exploration, Hungarian Acad. Sci., vol. I, pp. 75-84.

Rickman, H., and Huebner, W.F. (1990) 'Comet Formation and Evolution,' in W.F. Huebner (ed.), Physics and Chemistry of Comets, Springer Verlag, in press.

Rickman, H., Froeschlé, C., and Klinger, J. (1985) 'Amorphous-Crystalline Phase Transition and the Light Curve of Comet P/Halley,' in J. Klinger, D. Benest, A. Dollfus, and R. Smoluchowski (eds.), Ices in the Solar System, NATO ASI Series C, vol. 156, pp. 419-428.

Rickman, H., Fernández, J.A., and Gustafson, B.A.S. (1990) 'Formation of Stable Dust Mantles on Short-Period Comet Nuclei,' Astron. Astrophys., in press.

Sagdeev, R.Z., Elyasberg, P.E., and Moroz, V.I. (1988) 'Is the Nucleus of Comet Halley a Low Density Body?,' Nature 331, 240-242.

Schmitt, B. (1986) 'La surface de la glace: Structure, dynamique et interactions. Implications astrophysiques,' Doctoral thesis, Univ. of Grenoble.

Schmitt, B., and Klinger, J. (1987) 'Different Trapping Mechanisms of Gases by Water Ice and Their Relevance for Comet Nuclei,' in E.J. Rolfe and B. Battrick (eds.), Diversity and Similarity of Comets, ESA SP-278, pp. 613-619.

Schmitt, B., Grim, R.J.A., and Greenberg, J.M. (1988a) 'Molecular Diffusion in Ices Implications for the Composition of Interstellar Grain Mantles and Comet Nuclei,' in E. Bussoletti, C. Fusco, and G. Largo (eds.), Experiments on Cosmic Dust Analogues, Kluwer Acad. Publ., pp. 259-269.

Schmitt, B., Espinasse, S., Klinger, J., and Greenberg, J.M. (1988b) 'Effet de la température sur l'évolution et la différenciation chimique des glaces cométaires,' in M.C. Festou and D. Chabod (eds.), Comptes Rendus des Journées de Planétologie (CNRS-INSU), Observatoire de Besançon, pp. 203-206.

Schmitt, B., Espinasse, S., Grim, R.J.A., Greenberg, J.M., and Klinger, J. (1989) 'Laboratory Studies of Cometary Ice Analogues', in J. Hunt and T.D. Guyenne (eds.), Physics and Mechanics of Cometary Materials, ESA SP-302, pp. 65-69.

Shul'man, L.M. (1972) 'The Evolution of Cometary Nuclei,' in G.A. Chebotarev, E.I. Kazimirchak-Polonskaya, and B.G. Marsden (eds.), The Motion, Evolution of Orbits, and Origin of Comets, D. Reidel Publ. Co., Dordrecht, pp. 271-276.

Shul'man, L.M. (1983) 'Composition of Cometary Nuclei. A Cosmogonical Approach,' Acad. Sci. USSR Space Res. Inst., prepr. 771, 19 pages (in Russian).

Smoluchowski, R. (1981) 'Amorphous Ice and the Behavior of Cometary Nuclei,' Astrophys. J. 244, L31-L34.

Smoluchowski, R. (1982) 'Heat Transport in Porous Cometary Nuclei,' J. Geophys. Res. 87, Supp. A, 422 . 
Smoluchowski, R. (1985) 'Amorphous and Porous Ices in Cometary Nuclei,' in J. Klinger, D. Benest, A. Dollfus, and R. Smoluchowski (eds.), Ices in the Solar System, NATO ASI Series C, vol. 156, pp. 397-406.

Spohn, T., Benkhoff, J., Klinger, J., Grün, E., and Kochan, H. (1989) 'Thermal Modelling of Two KOSI Comet Nucleus Simulation Experiments,' Adv. Space Res. 9, No. 3, pp. 127-131.

Stern, S.A. (1988) 'Collisions in the Oort Cloud,' Icarus 73, 499-507.

Stern, S.A., and Shull, J.M. (1988) 'The Influence of Supernovae and Passing Stars on Comets in the Oort Cloud,' Nature 332, 407-411.

Strazzulla, G., Calcagno, L., and Foti, G. (1983) 'Polymerization Induced on Interstellar Grains by Low-Energy Cosmic Rays,' Mon. Not. R. Astr. Soc. 204, 59P-62P.

Tauber, F., and Kührt, E. (1987) 'Thermal Stresses in Cometary Nuclei,' Icarus 69, 83-90. Wallis, M.K. (1980) 'Radiogenic Melting of Primordial Comet Interiors,' Nature 284, 431433.

Wallis, M.K., and MacPherson, A.K. (1981) 'On the Outgassing and Jet Thrust of Snowball Comets,' Astron. Astrophys. 98, 45-49.

Weaver, H.A. (1989) 'The Volatile Composition of Comets,' ST SCI Prepr. No. 316.

Weissman, P.R. (1980) 'Stellar Perturbations of the Cometary Cloud,' Nature 288, 242-243.

Weissman, P.R. (1986a) 'How Pristine are Cometary Nuclei?,' in O. Melita (ed.), Comet Nucleus Sample Return, ESA SP-249, pp. 15-25.

Weissman, P.R. (1986b) 'Are Cometary Nuclei Primordial Rubble Piles?', Nature 288, 242243.

Weissman, P.R., and Stern, S.A. (1990) 'Physical Processing of Cometary Nuclei,' in Proc. of the workshop on Analysis of Returned Comet Nucleus Samples, Milpitas, CA, in press.

Whipple, F.L. (1950) 'A Comet Model. I. The Acceleration of Comet Encke', Astrophys. J. 111, 375-394.

Whipple, F.L., and Stefanik, R.P. (1966) 'On the Physics and Splitting of Cometary Nuclei,' Mém. Soc. R. Sci. Liège 12, 33-52.

Wood, J.A. (1987) 'Rosetta: A Mission to Sample the Nucleus of a Comet,' in E.J. Rolfe and B. Battrick (eds.), Diversity and Similarity of Comets, ESA SP-278, pp. 531-537.

Yabushita, S., and Wada, K. (1988) 'Radioactive Heating and Layered Structure of Cometary Nuclei,' Earth, Moon, Planets 40, 303-313.

Yamamoto, T. (1985) 'Formation Environment of Cometary Nuclei in the Primordial Solar Nebula,' Astron. Astrophys. 142, 31-36.

Yamamoto, T., and Kozasa, T. (1988) 'The Cometary Nucleus as an Aggregate of Planetesimals,' Icarus 75, 540-551.

Yamamoto, T., Nakagawa, N., and Fukui, Y. (1983) 'The Chemical Composition and Thermal History of the Ice of a Cometary Nucleus,' Astron. Astrophys. 122, 171-176. 